\title{
Proteogenomics integrating novel junction peptide identification strategy discovers three novel protein isoforms of human NHSL1 and EEF1B2
}

Cuitong $\mathrm{He}^{1,2}$, Jiangtao $\mathrm{Guo}^{2}$, Wenmin Tian²,3, and Catherine C. L. Wong ${ }^{1,2,3,4,5 *}$

${ }^{1}$ Peking-Tsinghua Centre for Life Sciences, Academy for Advanced Interdisciplinary Studies, Peking University, 100871, Beijing, China

${ }^{2}$ Center for Precision Medicine Multi-Omics Research, Peking University Health Science Center, 100191, Beijing, China

${ }^{3}$ School of Basic Medical Sciences, Peking University Health Science Center, 100191, Beijing, China

${ }^{4}$ Peking University First Hospital, 100034, Beijing, China

${ }^{5}$ Advanced Innovation Center for Human Brain Protection, Capital Medical University, 100069, Beijing, China

\section{Corresponding Authors}

Catherine C. L. Wong, E-mail: catherine_wong@bjmu.edu.cn.

Supporting information files: Figure S1-9\&Table S1-5 


\begin{tabular}{ll}
\hline Name & Supplementary Figure/Table Legends \\
\hline Figure S-1 & Evaluation of the effect of CJunction to enumerate junction peptides in MS data. "Highest scoring peptide" \\
& means only the top scoring peptide is taken into account for de novo sequencing, while "all candidate \\
peptides" means that all candidate peptides from de novo sequencing with different parameters are used & for the enumeration of junction peptides. "Percentage" refers to the number of junction peptides \\
& enumerated by CJunction as a percentage of the total number of annotated junction peptides co-identified \\
& by MaxQuant and pFind in the test dataset.
\end{tabular}

Figure S-2 Quality control of candidate novel peptides. (A) Box plot of the score of PSM within and beyond 1\% FDR. (B) Density distribution of the cosine similarity score between experimental and predicted spectra of candidate novel peptides. (C) Density plot of mass deviation of PSM identified at 1\% FDR at peptide level. (D) Density plot of the number of bases between adjacent CDSs (taken as the logarithm of the base 2) in human annotated protein isoforms.

Figure S-3 Validation of the novel peptide "CAGDIFGELEGQAAALGHR". (A) Raw score of the top 10 peptide candidates for the original spectrum of novel peptide. The raw score is a score given by pFind to characterize the PSM quality. (B) The five highest scoring comparisons obtained after NCBI BLASTP analysis of the novel peptides.

Figure S-4 Validation of the novel junction peptide "VKIPVSNLDEESR". (A) Comparison of the original and synthetic spectra of the identified novel junction peptide "VKIPVSNLDEESR". (B) Raw score of the top 10 peptide candidates for the original spectrum of novel peptide. The raw score is a score given by pFind to characterize the PSM quality. (C) The five highest scoring comparisons obtained after NCBI BLASTP analysis of the novel peptides.

Figure S-5 Localization of the coding sequence of the novel peptide "CAGDIFGELEGQAAALGHR" on chromosome VI and annotated protein isoforms of gene NHSL1. The small red square represents the position of the coding sequence of the novel peptide on the genome. This figure shows 16 annotated protein isoforms of NHSL1. Different colored squares represent different CDSs separated by introns, while squares with the same color represent the same CDS.

Figure S-6 The novel peptide "CAGDIFGELEGQAAALGHR" is widespread in annotated NHSL1 protein isoforms in other species. (A) Terminator to terminator region within the reading frame where the novel peptide is located. The N-terminal sequence containing the novel peptide is underlined. The novel peptide and the translation start codon ATG are marked in red. (B) Sequence alignment of the novel N-terminal in Figure A with annotated NHSL1 protein isoforms in other species. The "*” symbol indicates that the amino acids at this site are identical in multiple alignment sequences.

Figure S-7 Phylogenetic tree of two novel protein isoforms of human NHSL1.

Figure S-8 Validation of the novel peptide "SLSAAPQLSDTADTMGFGDLK". (A) Raw score of the top 10 peptide candidates for the original spectrum of the novel peptide. (B) The five highest scoring comparisons obtained after NCBI BLASTP analysis of the novel peptides.

Figure S-9 Homologous proteins of the novel protein isoform, elongation factor 1-beta isoform X2, are widespread in humans and many other species. The different colored squares represent different amino acid sequences. Squares with the same color represent similar amino acid sequences. In regions where Homo sapiens elongation factor 1-beta isoform X2 overlaps with other homologous proteins with high similarity (red and blue squares), white rectangles in the squares indicate unmatched amino acid sites.

Table S-1 (XLSX) FDRs at the PSM, peptide, and protein levels.

Table S-2 (XLSX) Universal Spectrum Identifiers for spectra of the three novel peptides.

Table S-3 (XLSX) CDS and amino acid sequence of NHS-like protein 1 isoform X15.

Table S-4 (XLSX) CDS and amino acid sequence of NHS-like protein 1 isoform X16.

Table S-5 (XLSX) CDS and amino acid sequence of elongation factor 1-beta isoform X2. 


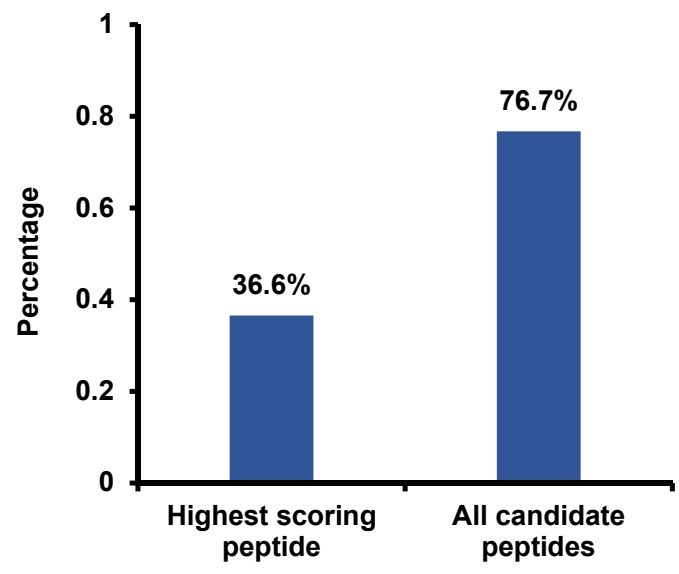

Figure S-1. Evaluation of the effect of CJunction to enumerate junction peptides in MS data. "Highest scoring peptide" means only the top scoring peptide is taken into account for de novo sequencing, while "all candidate peptides" means that all candidate peptides from de novo sequencing with different parameters are used for the enumeration of junction peptides. "Percentage" refers to the number of junction peptides enumerated by CJunction as a percentage of the total number of annotated junction peptides co-identified by MaxQuant and pFind in the test dataset. 
A

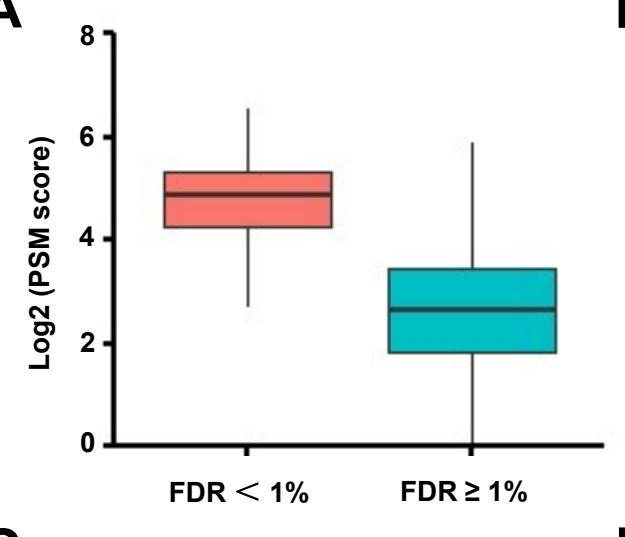

C

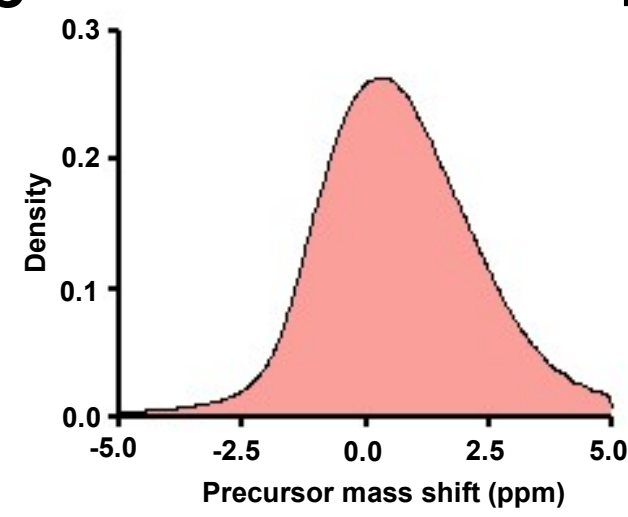

B

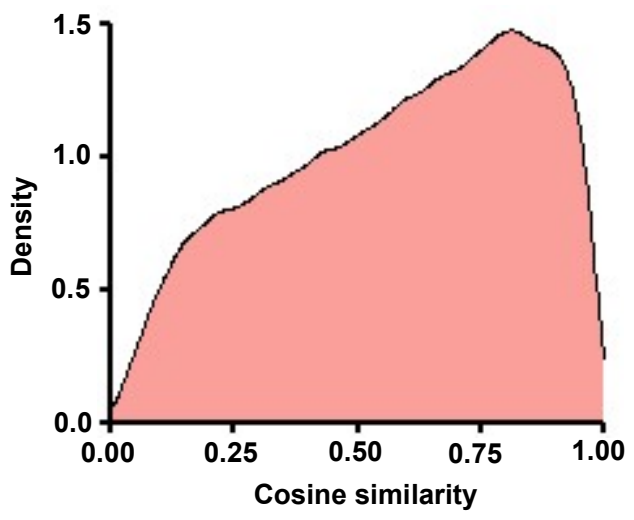

D

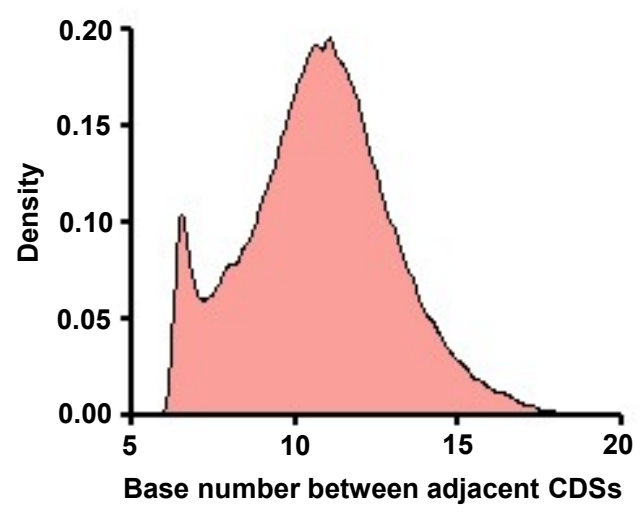

Figure S-2. Quality control of candidate novel peptides. (A) Box plot of the score of PSM within and beyond 1\% FDR. (B) Density distribution of the cosine similarity score between experimental and predicted spectra of candidate novel peptides. (C) Density plot of mass deviation of PSM identified at $1 \%$ FDR at peptide level. (D) Density plot of the number of bases between adjacent CDSs (taken as the logarithm of the base 2) in human annotated protein isoforms. 


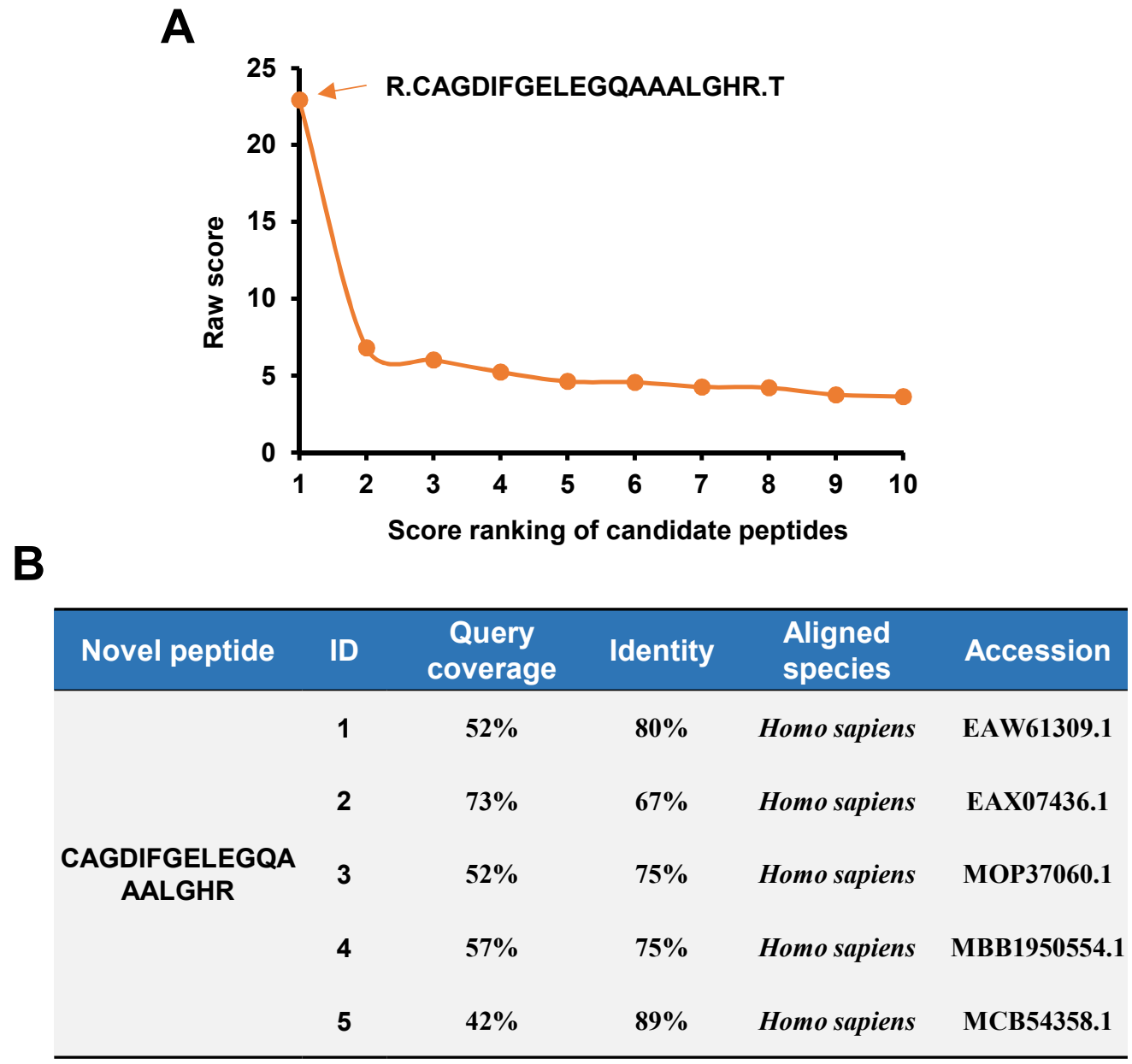

Figure S-3. Validation of the novel peptide "CAGDIFGELEGQAAALGHR". (A) Raw score of the top 10 peptide candidates for the original spectrum of novel peptide. The raw score is a score given by pFind to characterize the PSM quality. (B) The five highest scoring comparisons obtained after NCBI BLASTP analysis of the novel peptides. 
A

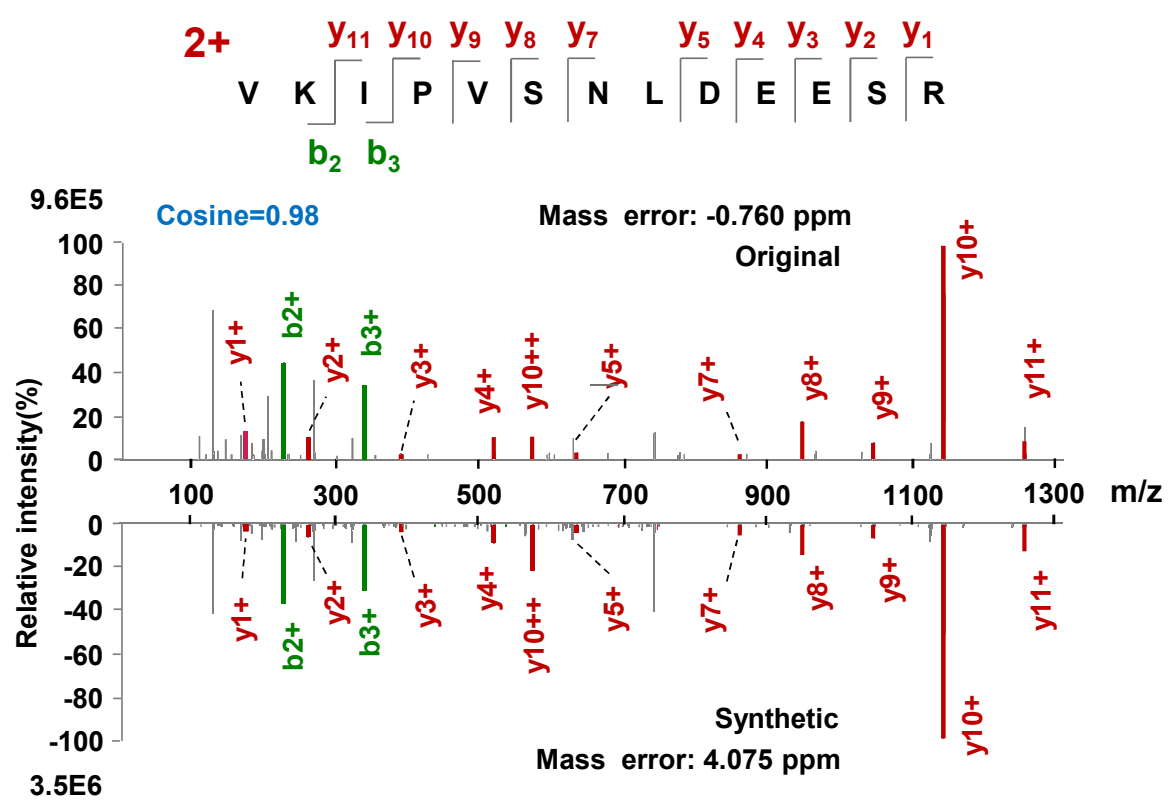

B

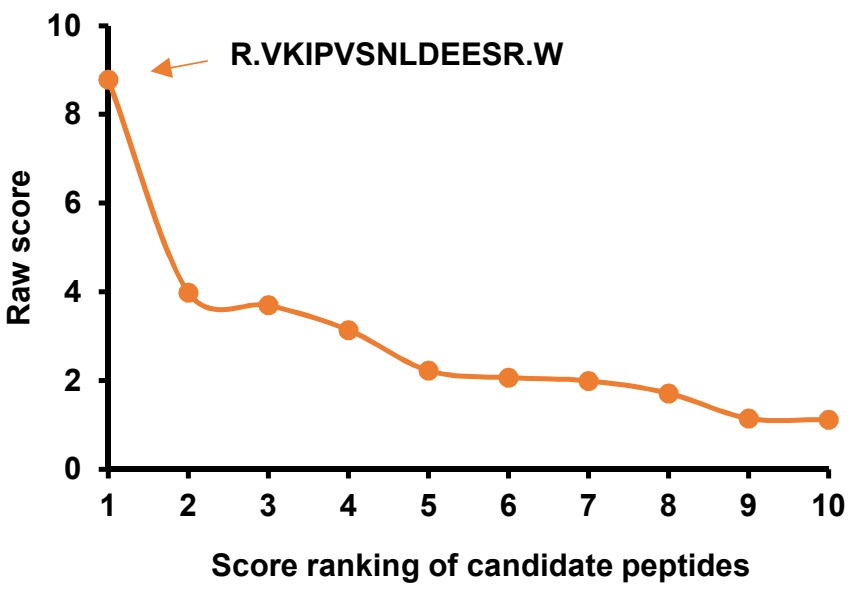

C

\begin{tabular}{|cccccc}
\hline Novel peptide & ID & $\begin{array}{c}\text { Query } \\
\text { coverage }\end{array}$ & Identity & $\begin{array}{c}\text { Aligned } \\
\text { species }\end{array}$ & Accession \\
\hline & 1 & $76 \%$ & $100 \%$ & Homo sapiens & XP_016866576.1 \\
& 2 & $69 \%$ & $100 \%$ & Homo sapiens & EAW47915.1 \\
VKIPVSNLDEESR & 3 & $100 \%$ & $69 \%$ & Homo sapiens & XP_011508956.1 \\
& 4 & $84 \%$ & $69 \%$ & Homo sapiens & AAY24159.1 \\
& 5 & $76 \%$ & $80 \%$ & Homo sapiens & ADN85614.1 \\
\hline
\end{tabular}


Figure S-4. Validation of the novel junction peptide "VKIPVSNLDEESR". (A) Comparison of the original and synthetic spectra of the identified novel junction peptide "VKIPVSNLDEESR". (B) Raw score of the top 10 peptide candidates for the original spectrum of novel peptide. The raw score is a score given by pFind to characterize the PSM quality. (C) The five highest scoring comparisons obtained after NCBI BLASTP analysis of the novel peptides. 


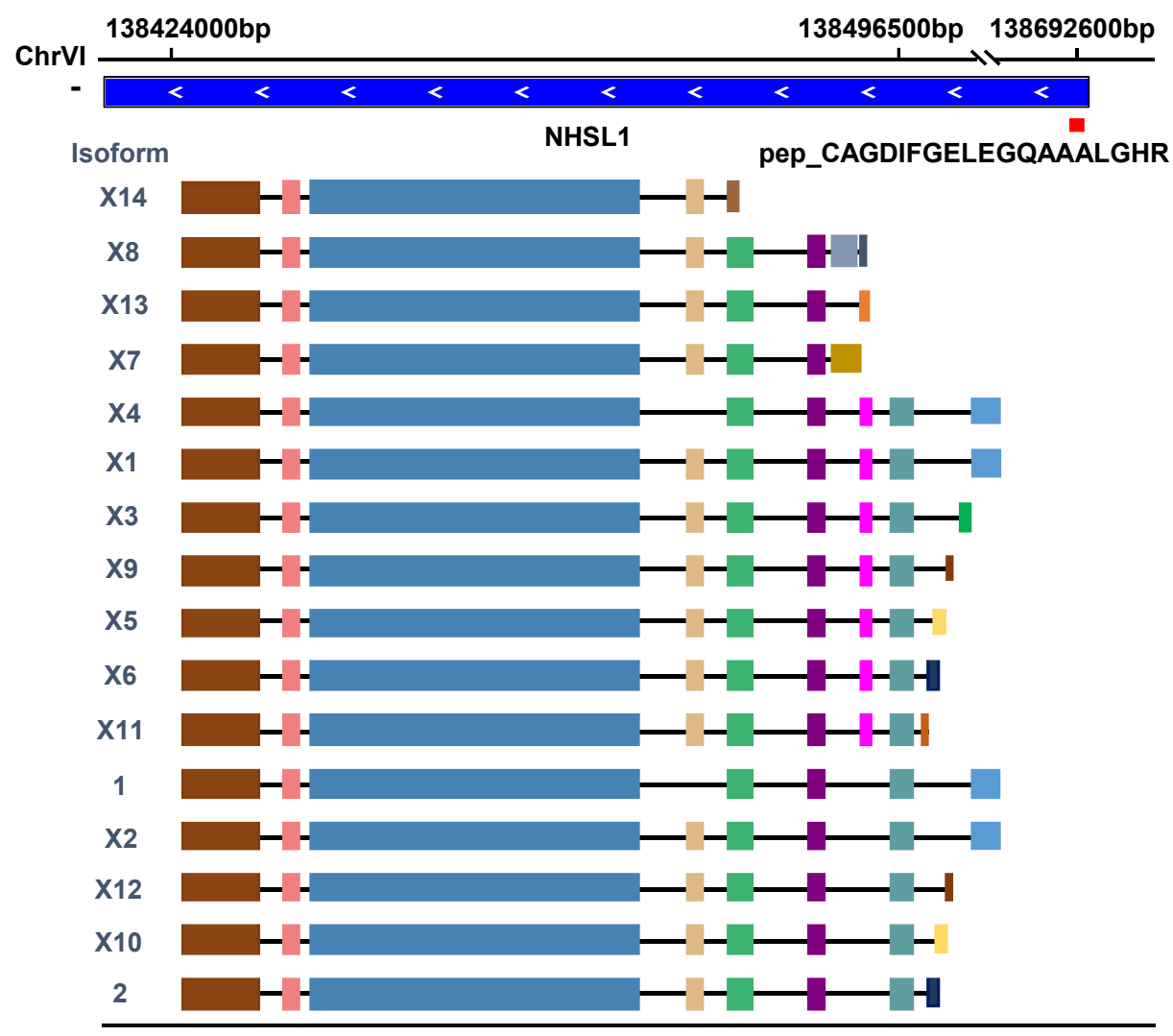

Figure S-5. Localization of the coding sequence of the novel peptide "CAGDIFGELEGQAAALGHR" on chromosome VI and annotated protein isoforms of gene NHSL1. The small red square represents the position of the coding sequence of the novel peptide on the genome. This figure shows 16 annotated protein isoforms of NHSL1. Different colored squares represent different CDSs separated by introns, while squares with the same color represent the same CDS. 
A

1 TGAGGGATCCCGCGCCGCCGGCCCGCCCCCGCCATGCCCTTCCACCAGAGGACCCTGGAG

$\begin{array}{lllllllllllllllllllll}1 & * & \mathrm{G} & \mathrm{I} & \mathrm{P} & \mathrm{R} & \mathrm{R} & \mathrm{R} & \mathrm{P} & \mathrm{A} & \mathrm{P} & \mathrm{A} & \mathrm{M} & \mathrm{P} & \mathrm{F} & \mathrm{H} & \mathrm{Q} & \mathrm{R} & \mathrm{T} & \mathrm{L} & \mathrm{E}\end{array}$

61 CCCGCGCGGCTGCGCCGGCCCGAGGCGGCGGGGGCCGGGGCTGCGGGCGCGCCACTCTTC

\begin{tabular}{lllllllllllllllllllll}
21 & $P$ & $A$ & $R$ & L & $R$ & $R$ & $P$ & $E$ & $A$ & $A$ & $G$ & $A$ & $G$ & $A$ & $A$ & $G$ & $A$ & $P$ & $L$ & $F$ \\
\hline
\end{tabular}

121 CGCTCGCTGGAGCAGGTCAGCTCGCATGCCCTGGGCTGCCTGCTGGCGCAGCTGGCCGAC

\begin{tabular}{lllllllllllllllllllll}
41 & $\mathrm{R}$ & $\mathrm{S}$ & $\mathrm{L}$ & $\mathrm{E}$ & $\mathrm{Q}$ & $\mathrm{V}$ & $\mathrm{S}$ & $\mathrm{S}$ & $\mathrm{H}$ & $\mathrm{A}$ & $\mathrm{L}$ & $\mathrm{G}$ & $\mathrm{C}$ & $\mathrm{L}$ & $\mathrm{L}$ & $\mathrm{A}$ & $\mathrm{Q}$ & $\mathrm{L}$ & $\mathrm{A}$ & $\mathrm{D}$ \\
\hline
\end{tabular}

181 CTGTCGCGCTGCGCCGGGGACATCTTCGGCGAGCTCGAGGGCCAGGCGGCCGCGCTGGGC

\begin{tabular}{lllllllllllllllllllll}
61 & $\mathrm{~L}$ & $\mathrm{~S}$ & $\mathrm{R}$ & $\mathrm{C}$ & $\mathrm{A}$ & $\mathrm{G}$ & $\mathrm{D}$ & $\mathrm{I}$ & $\mathrm{F}$ & $\mathrm{G}$ & $\mathrm{E}$ & $\mathrm{L}$ & $\mathrm{E}$ & $\mathrm{G}$ & $\mathrm{Q}$ & $\mathrm{A}$ & $\mathrm{A}$ & $\mathrm{A}$ & $\mathrm{L}$ & $\mathrm{G}$ \\
\hline
\end{tabular}

241 CACCGCACCGCCGCGCTGCACCGCCGCCTCGACGCCCTGCAAGCCGCCGCTGCGCGCCTG

$\begin{array}{lllllllllllllllllllll}81 & \mathrm{H} & \mathrm{R} & \mathrm{T} & \mathrm{A} & \mathrm{A} & \mathrm{L} & \mathrm{H} & \mathrm{R} & \mathrm{R} & \mathrm{L} & \mathrm{D} & \mathrm{A} & \mathrm{L} & \mathrm{Q} & \mathrm{A} & \mathrm{A} & \mathrm{A} & \mathrm{A} & \mathrm{R} & \mathrm{L}\end{array}$

301 GACCACCGCCGAGTGAAAATCCGTGAGTGGCCGGCGGGCCGGGGGAGGGGAACCGGCGGG

$\begin{array}{lllllllllllllllllllll}101 & D & H & R & R & V & K & I & R & E & W & P & A & G & R & G & R & G & T & G & G\end{array}$

361 GGAGGCACCCGGAGAGCTGCTCCCGCCCGCCCCCTGGCGGCCCTGGGTCGGCGCGGGGGC

$\begin{array}{lllllllllllllllllllll}121 & G & G & T & R & R & A & A & P & A & R & P & \text { L } & A & A & \text { L } & G & R & R & G & G\end{array}$

421 CGCGGAGCCCCGCGATGTCGGGGTCTCCAGTGGGGACTGCCGGACCCCAGCGGGAGGCTG

$\begin{array}{lllllllllllllllllllll}141 & R & G & A & P & R & C & R & G & L & Q & W & G & L & P & D & P & S & G & R & L\end{array}$

481 GGCAGGTGGCAGGGGCGCAGAGCGCTCTGTGACTTTCGGACTCCTGGAGGGTCTTGGCTA

$\begin{array}{lllllllllllllllllllll}161 & G & R & W & \text { Q } & G & R & R & A & \text { L } & \text { C } & \text { D } & \text { F } & \text { R } & \text { T } & \text { P } & \text { G } & \text { G } & \text { S } & \text { W } & \text { L }\end{array}$

541 CCTCGAGAGAGTGGGCGCGGAGGGTGGGAATTGGACAGTCCCCTTGTCTGCTCCTTCTGT

$\begin{array}{lllllllllllllllllllll}181 & \text { P } & \text { R } & \text { E } & \text { S } & \text { G } & \text { R } & \text { G } & \text { G } & \text { W } & \text { E } & \text { L } & \text { D } & \text { S } & \text { P } & \text { L } & \text { V } & \text { C } & \text { S } & \text { F } & \text { C }\end{array}$

601 CTGTCTGTGTCTGTCTGTTTGGGAGACCCCGGGGCGCTGTAG

$\begin{array}{lllllllllllllll}201 & \text { L } & \text { S } & \text { V } & \text { S } & \text { V } & \text { C } & \text { L } & \text { G } & \text { D } & \text { P } & \text { G } & \text { A } & \text { L } & *\end{array}$

B

******* $\quad * * * * * * * * \quad * * * \quad * * * * * * * * * * * * * * * * * * * * * * * * * * * * * * * * * * * * * * * * * * * * *$ MPFHQRTLEPARLRRPE-AAGAGAAGAPLFRSLEQVSSHALGCLLAQLADLSRCAGDIFGELEGQAAALGHR

Sapajus paella

NHS-like protein 1 isoform X1

Macaca fascicularis

NHS-like protein 1 isoform X1

Microtus ochrogaster

NHS-like protein 1 isoform $\mathrm{X} 1$

Rattus norvegicus

NHS-like protein 1 isoform X1

Mastomys coucha

NHS-like protein 1 isoform X1
MPFHQRTLEPARLRRPE-AAGAGAAGAPLFRSLEQVSSHALGCLLAQLADLSRCAGDIFGELEGQAAALGHR

MPFHQRTLEPARLRRPV-AAGAGAAGAPLFRSLEQVSSHTLGCLLAQLADLSRCAGDIFGELEGQAAALGHR

MPFHQRSVEPARLRRPEETAVARAAGAPLFRSLEQVSSHTLVCLLAQLADLSRCAGDIFGELEGQAAALGHR

MPFHQRSVEPARLRRPEEAAGALAGDPPLFRSLEQVSSHTLVCLLAQLADLSRCAGDIFGELEGQAAALGHR

MPFHQRSVEPARLRRPEEAAPARAGDPPLFRSLEQVSSHTLVCLLAQLADLSRCAGDIFGELEGQAAALGHR 
Figure S-6. The novel peptide "CAGDIFGELEGQAAALGHR" is widespread in annotated NHSL1 protein isoforms in other species. (A) Terminator to terminator region within the reading frame where the novel peptide is located. The N-terminal sequence containing the novel peptide is underlined. The novel peptide and the translation start codon ATG are marked in red. (B) Sequence alignment of the novel N-terminal in Figure A with annotated NHSL1 protein isoforms in other species. The "*" symbol indicates that the amino acids at this site are identical in multiple alignment sequences. 


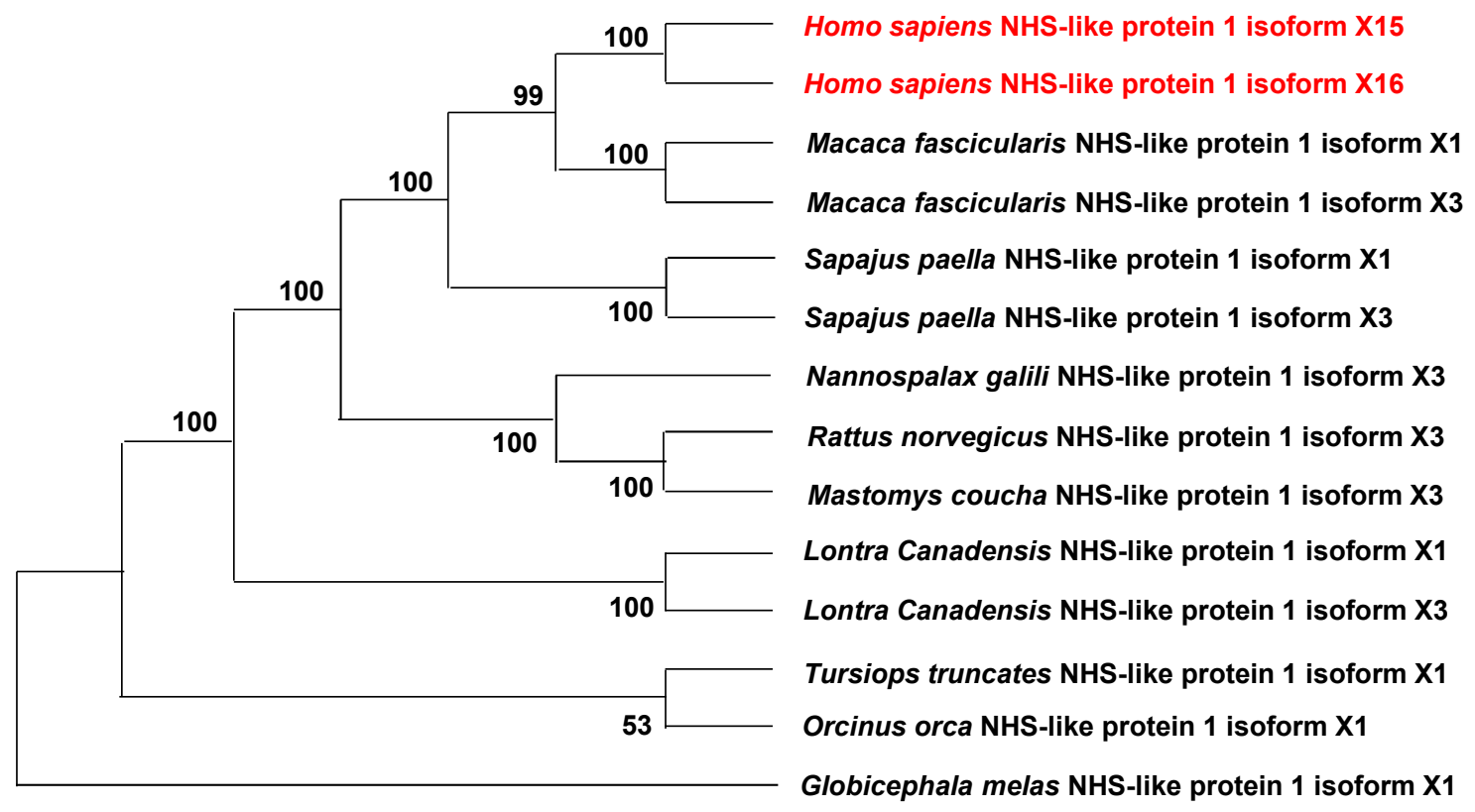

Figure S-7. Phylogenetic tree of two novel protein isoforms of human NHSL1. 


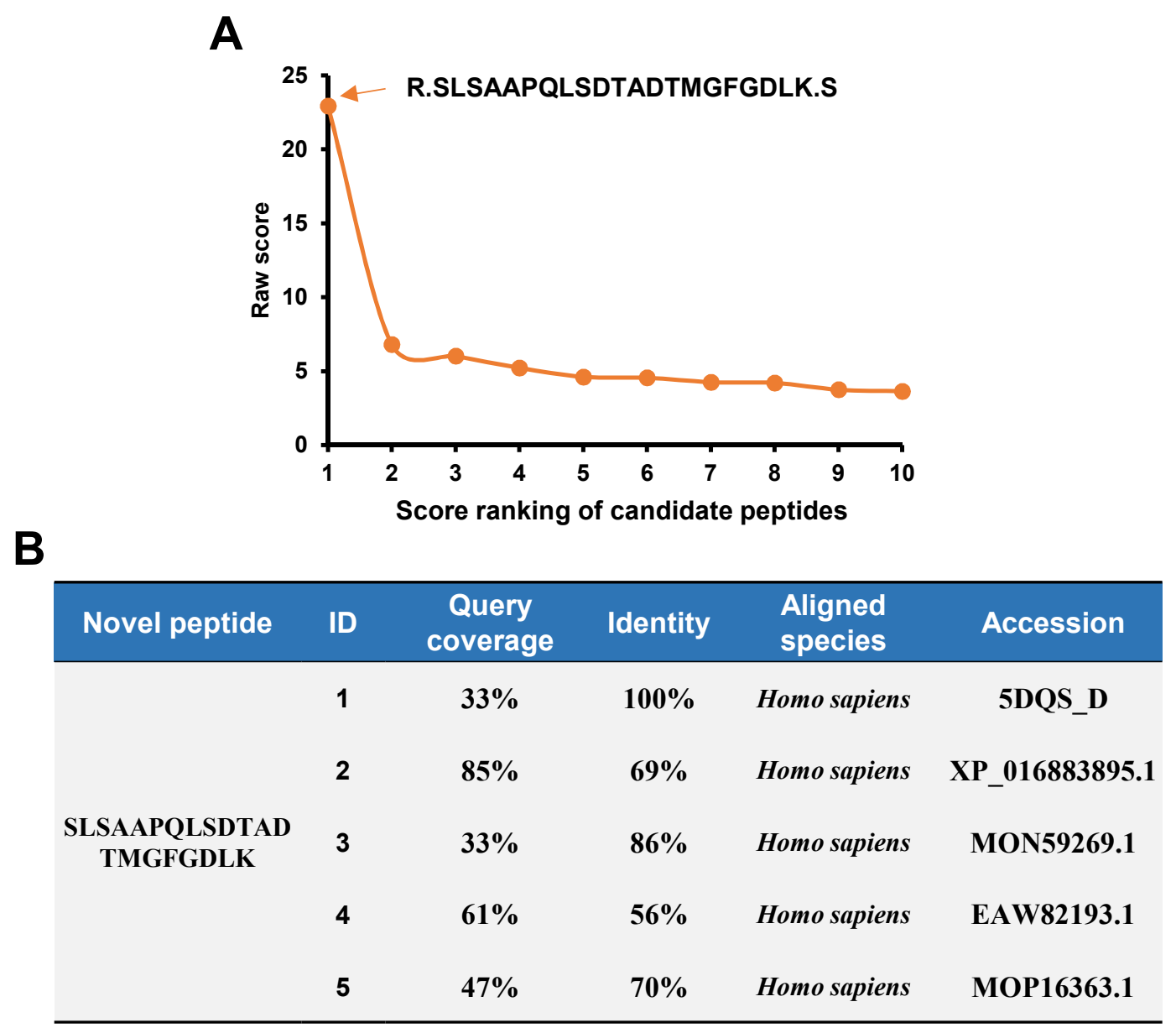

Figure S-8. Validation of the novel peptide "SLSAAPQLSDTADTMGFGDLK". (A) Raw score of the top 10 peptide candidates for the original spectrum of the novel peptide. (B) The five highest scoring comparisons obtained after NCBI BLASTP analysis of the novel peptides. 


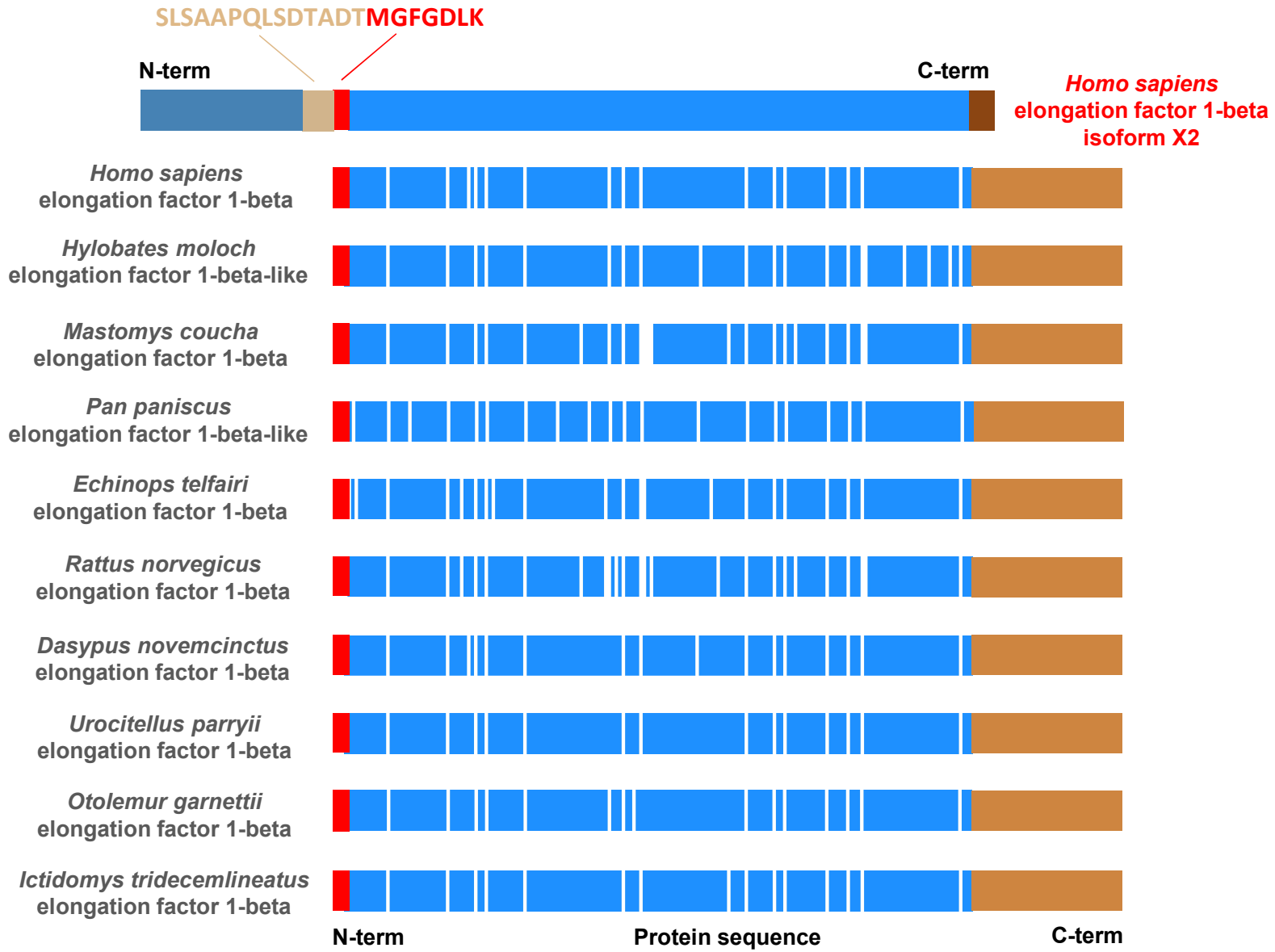

Figure S-9. Homologous proteins of the novel protein isoform, elongation factor 1-beta isoform X2, are widespread in humans and many other species. The different colored squares represent different amino acid sequences. Squares with the same color represent similar amino acid sequences. In regions where Homo sapiens elongation factor 1-beta isoform X2 overlaps with other homologous proteins with high similarity (red and blue squares), white rectangles in the squares indicate unmatched amino acid sites. 